\title{
Planning Image-Based Measurements in Wind Tunnels by Virtual Imaging
}

\author{
Laura K. Kushner* \\ ACI/NASA Ames Research Center, Moffett Field, CA, 94035, USA \\ Edward T. Schairer ${ }^{\dagger}$ \\ NASA Ames Research Center, Moffett Field, CA, 94035, USA
}

\begin{abstract}
Virtual imaging is routinely used at NASA Ames Research Center to plan the placement of cameras and light sources for image-based measurements in production wind tunnel tests. Virtual imaging allows users to quickly and comprehensively model a given test situation, well before the test occurs, in order to verify that all optical testing requirements will be met. It allows optimization of the placement of cameras and light sources and leads to faster set-up times, thereby decreasing tunnel occupancy costs. This paper describes how virtual imaging was used to plan optical measurements for three tests in production wind tunnels at NASA Ames.
\end{abstract}

\section{Nomenclature}

$\psi \quad$ Rotor azimuthal angle, degrees, where $0^{\circ}$ is directly downstream

$\alpha \quad$ Angle of Attack, degrees

$\beta \quad$ Yaw, degrees

\section{Introduction}

Imaging-based measurements in wind tunnels have become commonplace in production tests due to advances in camera sensors, short-duration, high-power lights and computer technology. Commonly used technologies include pressure-sensitive paint (PSP) for measuring pressure distributions on the surfaces of models, particle image velocimtery (PIV) for measuring flow velocities, schlieren and background oriented schlieren (BOS) for measuring flow density variations, and photogrammetry for measuring model deformation and movement. Proper selection and placement of cameras and illumination sources is critical to the success of these imaging techniques. In most production wind tunnels the design of an imaging system is likely to be severely constrained by the positions of test-section windows relative to the model and the region of interest (ROI). As few wind tunnels were designed with imaging techniques other than schlieren in mind, windows are rarely optimally placed for other visual techniques. In addition to limiting camera locations, this can limit illumination, which for some techniques results in longer data acquisition times, reducing data throughput and increasing costs. Therefore, the design of an image-based measurement system can be a complicated task that involves many compromises.

To design an imaging system, it is best to know how resulting images from the cameras will look. Real images of the model installed in the test section, however, are unlikely to be available during the design process. High tunnel occupancy costs and tight schedules leave no time for trial and error-the system must be ready for installation when the test begins. Moreover, it is likely that a system will require special hardware, including mounting brackets, camera cooling lines, electrical cabling, and special camera lenses,

\footnotetext{
*Associate Engineer, ACI/Experimental Aero-Physics Branch, M/S 260-1, Non-Member.

${ }^{\dagger}$ Aerospace Engineer, Experimental Aero-Physics Branch, M/S 260-1, Non-Member.
} 
all of which depend on the layout of the system and must be purchased or fabricated in advance. In some cases it is even necessary to add windows to a test section which clearly must be designed well before the test begins.

One approach to designing an imaging system is to mock up the test section, model, and imaging system in a laboratory. This is expensive, time-consuming and impractical if the test section and model are large. Also, a physical mockup, even at reduced scale, requires a replica of the model, which is unlikely to exist and would be expensive to build. A much more economical and versatile solution is to use computer graphic simulation to predict virtual images of the test-section scene. This allows many imaging configurations to be examined quickly and inexpensively. Furthermore, computer graphic simulation can supply more complete information about the imaging configuration than would be possible with a physical mockup: virtual images can be computed using a high-fidelity representation of the model over the full range of model positions and attitudes; quantitative information about camera viewing angles, model illumination intensities, and scattered-light intensities can be extracted from the simulations; glare lines and specular reflections of laser sheets from polished model surfaces (which can severely damage cameras) can be predicted; and objects of interest (for example, a PIV calibration grid) may be effortlessly added to the scene. Clearly, numerical simulation can produce a better solution to the imaging problem at far lower cost than a physical mockup.

This paper describes how virtual imaging was used to plan three different optical measurement systems for three major tests in production wind tunnels at NASA Ames Research Center. In the first example, stereo photogrammetry and Retroreflective Background Oriented Schlieren (RBOS) were used to measure the positions of vortices shed by a UH-60 helicopter rotor in the Air Force National Full-Scale Aerodynamic Facility (NFAC) 40- by 80-Foot Wind Tunnel at NASA Ames; in the second example, PSP was used to measure the pressure distribution on the surface of a $6 \%$-scale model of the Orion Crew Exploration Vehicle (CEV) in the Unitary Plan 9- by 7- Foot Supersonic Wind Tunnel (9x7 SWT) and 11- by 11- Foot Transonic Wind Tunnel (11x11 TWT); and in the third example, PIV was used to measure flow velocities in the wake of a generic commercial transport aircraft in the 11x11 TWT.

\section{Virtual Imaging Software}

Virtual imaging software ${ }^{1}$ was developed at Ames Research Center to help plan three-component (3C) PIV measurements that were made during Return-to-Flight (RTF) tests of the Space Shuttle ascent configuration in the 9x7 SWT. ${ }^{2}$ This test was the first application of PIV in the 9x7 SWT where the windows, which were designed to accommodate schlieren measurements, severely constrained the design of the 3C PIV system. One option for analyzing this imaging problem was to use the Virtual Diagnostics Interface (ViDI), ${ }^{3}$ virtual-imaging software developed at NASA Langley Research Center. The start-up costs of using this software, however, were considerable and included a steep learning curve, purchasing expensive commercial animation graphics software upon which ViDI is based, and developing complex input data files to represent the wind tunnel. As an alternative, in-house software was developed using simple virtual-imaging algorithms that had already been developed to display PSP data. The software executed directly under Windows operating systems without any other underlying software or graphics packages. An important advantage of developing in-house software was that it could be tailored to meet needs as they arose.

The in-house software, dubbed "Vinci", was designed to compute images of wind-tunnel scenes using only the bare minimum of information necessary to represent features relevant to the imaging application. Therefore, the only required data file is a numerical description of the model. This can be a surface grid used for computational fluid dynamics (CFD), which is likely to already exist since many wind-tunnel tests are run in conjunction with CFD. A graphical user interface (GUI) allows the user to enter information about the positions and orientations of both the model and up to 20 cameras as well as characteristics of the camera sensors, such as pixel size and density, and lens focal lengths. The GUI then displays a computed, "virtual", image of the model from the perspective of one of the cameras. Currently, the format of the surface grid files must be either Plot3D, ${ }^{4}$ commonly used for CFD, or stereo-lithography.

Additional objects may be added to a scene by reading surface grids from one or more additional grid files, or the application itself can be used to create simple geometric shapes that can be combined to represent more complex objects. Vinci includes functions to automatically create a variety of special objects including: test-section walls and windows, laser sheets and calibration plates (for PIV and Doppler Global Velocimetry (DGV)), fiducial marks (applied to the model for PSP and photogrammetry), lamps (for PSP), and plane and convex mirrors (for photogrammetric recession measurements (PRM)). ${ }^{5}$ The cameras themselves, their 
fields of view (i.e., the expanding rectangular region in space extending outward from the camera that is imaged on the sensor), and the volume of space where the fields of view intersect may also be visualized from an arbitrary vantage point outside the test section.

In addition to images, the virtual-imaging software computes many parameters that are important for a variety of optical measurements. For example, for PIV and DGV measurements, where a plane in the flow is illuminated by a sheet of laser light and imaged from different directions by two or more cameras, Scheimpflug angles (the angle between the lens and the sensor plane), light scattering angles (angle between the incident ray and the vector from the scattering point to the camera), and relative scattered-light intensities (for Mie or Rayleigh scattering) are computed for each camera. Specular reflections due to the laser sheet striking the model can also be computed and displayed, thus identifying regions in the test section where cameras should not be placed. For pressure-sensitive paint (PSP) measurements, the viewing angle of each panel of the model as seen by each camera may be computed, and the panel may be color-coded, either by the angle to each camera or by the best (least oblique) angle of all cameras. Likewise, the intensity of illumination of each panel by PSP lamps may be computed and displayed. The application may also be used to project reduced data into the wind-tunnel scene, thereby putting the data in spatial context with the model. For example, for PIV and DGV, the laser sheet may be replaced with a color contour map showing the measured velocities in the plane, or, for PSP, each panel of the model may be color coded by the pressure measured at that panel.

Image coordinates of each node of all surface grids are computed from the space coordinates of the node using the Direct Linear Transformation (DLT) used in photogrammetry. ${ }^{6}$ The eleven coefficients of this transformation are defined for each camera by the position, pointing angles, lens focal length, and sensor of the camera. The virtual images account for hidden surfaces and shadows.

Vinci is still used at NASA Ames in spite of the proliferation of powerful graphics software that is available at little or no cost because of the ease with which objects and camera characteristics can be defined and capabilities can be added as needs arise.

\section{LRTA UH-60 Airloads Test}

A full-scale UH-60 Blackhawk helicopter rotor was tested on the Large Rotor Test Apparatus (LRTA) ${ }^{7}$ in the NFAC ${ }^{8}$ at NASA Ames Research Center. One of the objectives of this test was to measure the positions of vortices produced by the advancing blade. This was accomplished by simultaneously imaging a region of interest (ROI) just downstream of the advancing blade from two directions and visualizing vortices in both views by RBOS. The object-space coordinates of the vortices were then calculated from the image-plane coordinates using stereo photogrammetry.

\section{III.A. Retroreflective Background Oriented Schlieren}

Background Oriented Schlieren (BOS $)^{9}$ is an optical technique similar to schlieren and shadowgraphy that measures the deflection of light rays from an illumination source due to density gradients in the test section. It is used in situations where traditional schlieren techniques are not feasible due to optical constraints. A speckle pattern in the background of the region of interest is imaged in quiescent, wind-off conditions and also at wind-on test conditions. When a vortex filament lies in the foreground of a BOS background, the steep density gradient in the vortex core causes the speckled background in the neighborhood of the vortex to appear to shift in the direction of the decreasing density, i.e., toward the vortex core. This characteristic makes it possible to precisely locate vortices in the BOS images. These apparent shifts in the background are measured in the same way as particle displacements are measured in PIV images: points of interest in the images are defined by a rectangular grid, and the local image-plane displacement at each node of the grid due to density gradients is measured by cross correlating the wind-off and wind-on images in a small (e.g., $20 \times 20$ pixel) interrogation window centered on the node. The displacement of the peak of the correlation function from the center of the interrogation window measures the local displacement between the wind-off and wind-on images.

With Retroreflective Background Oriented Schlieren (RBOS), ${ }^{10}$ dark speckles are applied to a retroreflective background and the illumination source is placed with the camera, maximizing the retro-reflected light returned from the background. This increases the contrast between the dark speckles and bright background, improving the signal-to-noise ratio (SNR), which allows shorter integration times that can 
freeze flow unsteadiness. The only optical access that is needed is a small window that can accommodate both the camera and the light source. Therefore RBOS can provide schlieren-type data in wind tunnels that lack large, expensive, schlieren-quality windows.

\section{III.B. Stereo Photogrammetry}

In stereo photogrammetry, ${ }^{11}$ a region of interest is imaged from at least two directions. The space coordinates of a point in the ROI that appears in both images can be computed from the image-plane coordinates of the point in both images and camera calibration coefficients. The cameras can be calibrated in situ by placing in the ROI an object with targets whose spatial positions are known and imaging the object with both cameras. The sensitivity of the measurement in the direction perpendicular to the baseline between the cameras is greatest when the angular separation between the cameras is $90^{\circ}$.

One of the requirements of stereo photogrammetry is that measurement points in the images of both cameras must correspond to the same physical point in space. In many applications of photogrammetry, particularly those that use fiducial marks, this correspondence is self evident. In the present test, however, where there were no fiducial marks and the vortices were invisible, epipolar geometry, ${ }^{11,12}$ which does not require fiducials, was used to establish correspondence between points on the vortex in the images of both cameras.

\section{III.C. Wind Tunnel}

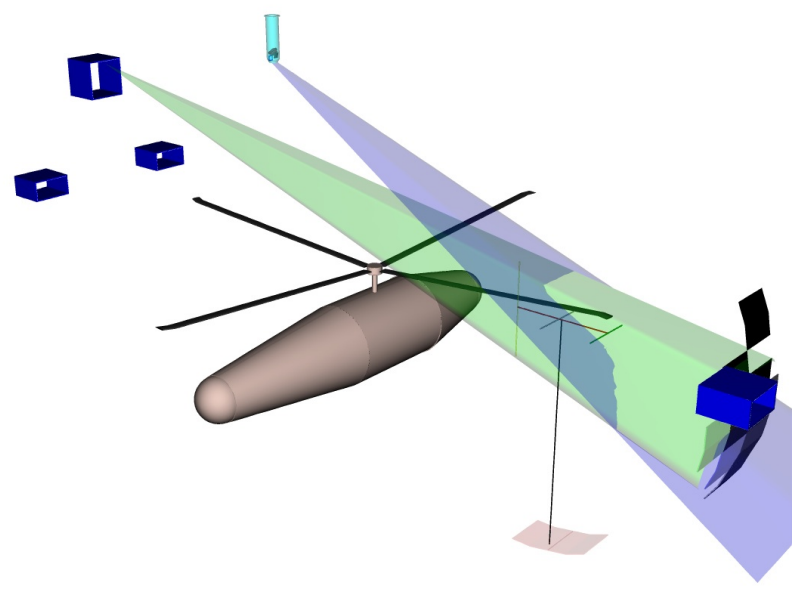

The 40- by 80 - Foot test section of the NFAC is oval-shaped in cross-section, the ceiling and floor are flat, the sidewalls are semi-circular. There are very few windows: three in each sidewall just above the centerline, and a new window in the port sidewall well above the centerline added for a 3C PIV system. In addition, temporary windows were added to the floor to accommodate cameras of an optical blade deflection measurement system for this test. The walls of the test section are lined with four- by four-foot acoustic panels that together create a semi-anechoic space for aeroacoustic research. Adding windows is undesirable because it is expensive and each window eliminates an acoustic panel, degrading the existing high-quality, low-noise flow in the tunnel.

The model and LRTA were mounted on a turntable in the floor of the test section. The rotor mast was just upstream of the new PIV window, and the rotor plane was midway between the floor and ceiling. The region of interest was just downstream of the advancing blade as it passed the $\psi=90^{\circ}$ position, on the starboard side of the model.

\section{III.D. Virtual Image Use for LRTA UH-60 Airloads RBOS}

RBOS was a natural choice to capture schlieren-like data in a test section with few windows. The challenge was to find two available windows from which the ROI could be imaged with sufficient separation to allow stereo measurements. In addition, the acoustic panels that lay in the background of the ROI as seen from each camera position would have to be covered with a suitable RBOS background. Because each RBOS background panel compromised the acoustic liner, it was desirable to have as much overlap of the backgrounds for the two cameras as possible so that fewer acoustic panels would need to be covered. The background panels also had to be accessible (i.e., not in the ceiling or very high in the sidewalls ) and could not be in a high-traffic area (e.g., on the floor of the test section). 


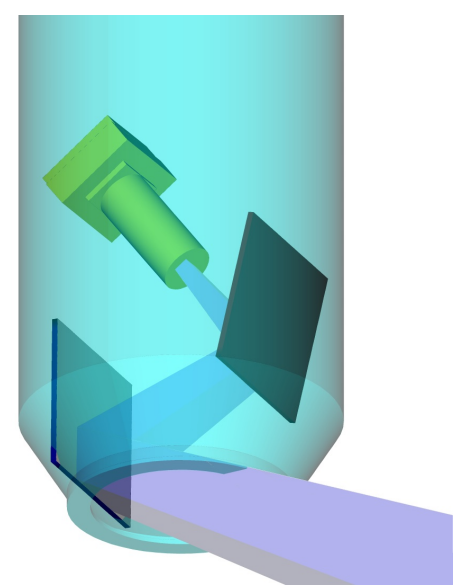

(a) Camera and mirrors only

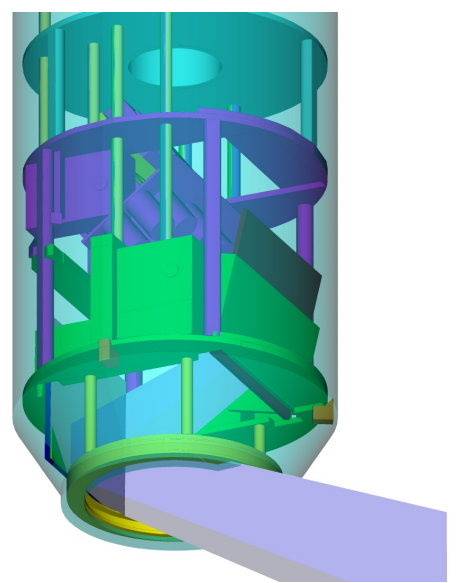

(b) Mounting hardware and optics

Figure 2. Virtual images of periscope.

In a previous proof-of-concept test of the same model, cameras were mounted in one of the sidewall windows on the port side of the test section, downstream of the model. In this position, however, the cameras viewed the ROI (on the opposite side of the test section) through vortices shed by the retreating blade, whose plane of rotation lay immediately under the window. The near-side vortex often obscured vortices in the ROI. To avoid this interference, both cameras needed to be located well above the rotor plane. The new PIV window in the port sidewall just downstream of the rotor mast was suitable for mounting one camera, but there was no properly situated window for the second camera. There were, however, light fixtures in the ceiling of the test section that could accommodate a camera if the lamp were removed. Therefore, the second camera was located in a light fixture slightly downstream of the sidewall camera on the port side of the tunnel centerline. Light fixtures on the starboard side of the centerline, closer to the ROI, were rejected because they would have required placing retro-reflective panels on the floor of the test section. The light fixtures are 68 inches deep and 11.5 inches in diameter, tapering to a 8 inch in diameter lens, the face of which forms a pressure seal with the test section. Because of the restricted space in the light fixture and the oblique, cross-stream viewing angle to the ROI, light needed to be directed to the ceiling-mounted camera by a periscope.

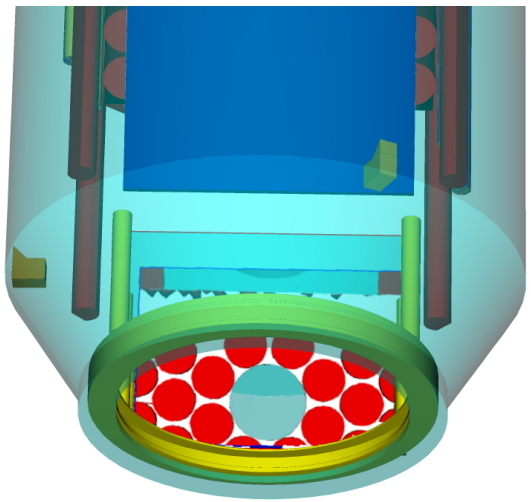

Figure 3. Looking backward along optical axis of the periscope. LEDs are red.
Virtual imaging and the ability to compute compound reflections were critical to the design of the periscope. The space in which the periscope was to be installed - at the bottom of a vertical cylindrical tube more than 5 feet deep, less than a foot in diameter, and 40 feet above the floor of the test section - was completely inaccessible, making direct visual observation of what could been seen from this position almost impossible. First, the camera (Redlake EC11000 with $9 \mu \mathrm{m}$ pitch pixels in a $4008 \times 2672$ array) and two mirrors at the bottom of the cylinder were modeled, centering the region of interest in the field of view (FOV) of the camera (Figure 2(a)). Next, a custom-built ring lamp consisting of 18 high-intensity red LED modules that surrounded the camera lens was added. Light from the LEDs was directed through the ROI and to the RBOS background by the periscope mirrors; and retro-reflected light from the background was directed to the camera by the same mirrors. The CAD-like functions in Vinci were then used to design and model mounting brackets and other structures for the mirrors, camera, lamp and window at the bottom of the periscope to verify that these structures fit together properly and did not obstruct either the camera or the lamps (Figure 2(b)). The structure included mechanisms for adjusting the angles of the camera and both mirrors from the top of the cylinder. Finally, a lens focal length $(200 \mathrm{~mm})$ was selected to fill the FOV with the ROI.

Although Vinci could compute reflections from the ROI as seen through the periscope, the capability 
to compute the reflected light from the ring lamp toward the ROI has not been implemented. Therefore, to verify that the mirrors were big enough to reflect light from all elements of the ring lamp and that the lamps were not blocked either by the window frame or by internal parts of the periscope, a virtual view was computed that looked backwards from the ROI towards the periscope along its optical axis. In this view, each separate LED of the ring lamp must be visible as a reflection in the periscope mirrors if light from the lamp is to reach the ROI. This imaging showed that at least a portion of each lamp element was visible through the window, but that the window frame obstructed large portions of elements furthest from the optical axis. It also showed that this obstruction could be reduced by beveling the window frame (Figure 3 on the preceding page).

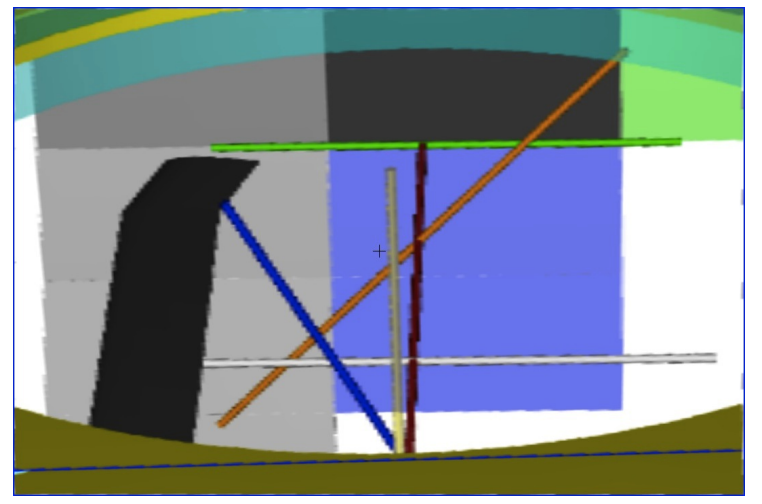

(a) Virtual Image, Periscope Camera.

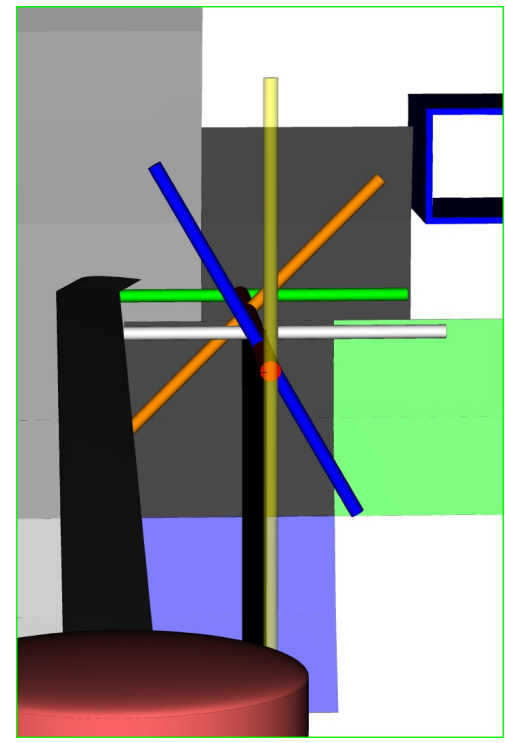

(c) Virtual Image, Side Wall Camera.

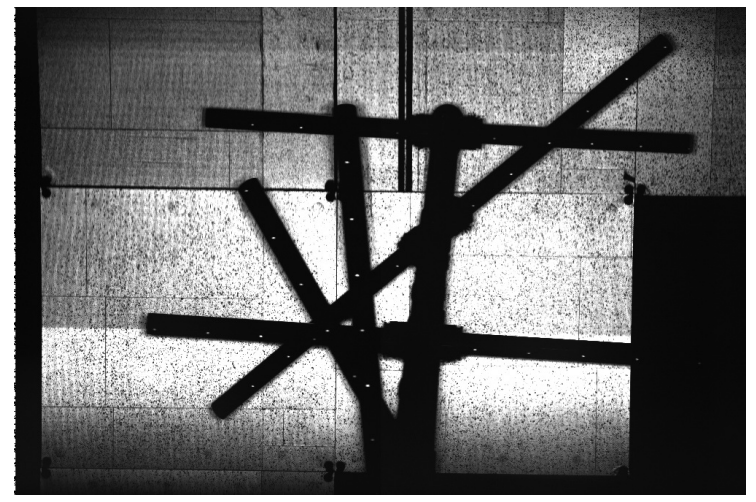

(b) Real Image, Periscope Camera.

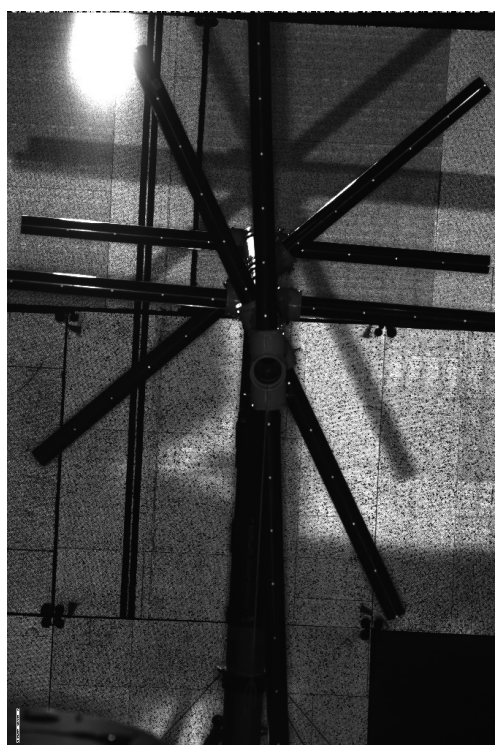

(d) Real Image, Side Wall Camera.

Figure 4. Virtual and actual images of RBOS camera fields of view, with calibration rig shown. Rotor was rotated out of FOV for real images to avoid interference with calibration rig.

Predicting the view of the second camera mounted in the port-side PIV window was much simpler because it viewed the ROI directly. The camera and lamp were the same as for the periscope except that a longer lens was required $(300 \mathrm{~mm})$ because of the greater distance to the ROI. Both cameras were synchronized to the rotor and each exposure lasted $40 \mu \mathrm{s}$, which corresponds to the time required for the blade tip to advance one chord-length (23.7 in). Both lamps and cameras were triggered by a once-pre-revolution signal from the rotor, with suitable delays to allow measurements at different azimuth angles of the rotor.

Once the camera FOVs were established, virtual imaging was used to identify acoustic panels in the background of each view. Eight panels filled the backgrounds of both cameras. Quarter-inch aluminum plating was cut to fit the shape of these $4 \mathrm{ft} \times 4 \mathrm{ft}$ panels. The plates were then covered with retroreflective sheeting, splattered with black ink and mounted over the acoustic panels in the background. Finally, virtual 
images were also used to design a calibration rig that spanned the overlapping fields of view. These images were then used to verify that targets on the rig were visible to both cameras and were the proper size for locating with target-finding software.

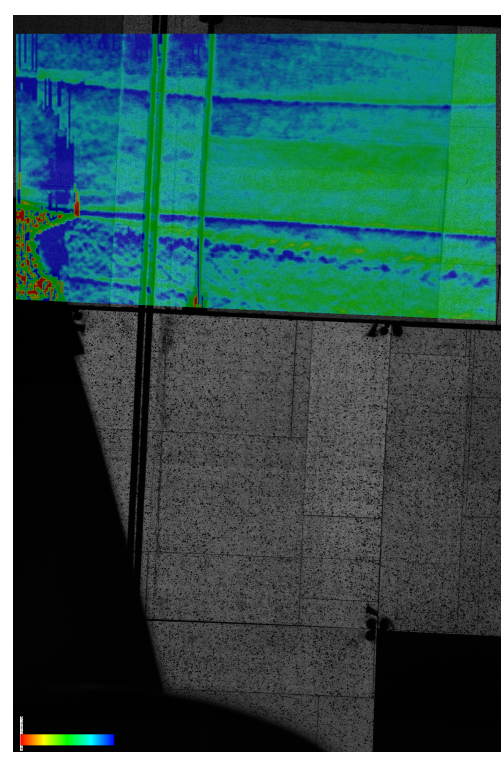

Figure 5. Image from the side-wall camera with image-plane displacement data overlay. Rotor is present on the left side of the image; multiple vortices are visible as blue and green pairs. and moments on the model and compared to the data from the balance, it was necessary for the PSP system to have full optical coverage of model, while not obstructing the collimated beam of the shadowgraphy system, which occupies a 4-foot diameter in the center of the window blank.

\section{IV.A. Pressure Sensitive Paint}

Pressure Sensitive Paint is an optical technique to measure continuous surface pressure distributions on wind tunnel models and flight vehicles. ${ }^{14}$ When excited by ultraviolet lamps, the paint fluoresces, and the strength of this luminescence is inversely proportional to the local surface pressure. In order to capture high-quality PSP data certain requirements must be met. The model must be evenly illuminated by UV lamps to avoid low SNR in more dimly lit regions where changes in luminescence are harder to detect. The goal was to illuminate all surfaces of the model to within two f-stops. In principal, PSP can acquire data on surfaces where the camera viewing angle is very oblique. Surfaces painted with PSP are approximately Lambertian, with intensity of luminescence independent of viewing angle. Spatial resolution, however, decreases with increasing angle so large viewing angles are undesirable. For the purposes of planning the layout of PSP cameras a viewing angle of $70^{\circ}$ (angle between a vector perpendicular to the local surface and the direction to the camera) was considered to be the upper limit of what was usable.

\section{IV.B. Wind Tunnel and Imaging System}

26-AA ran in two test sections of the Unitary Plan Wind Tunnel at NASA Ames. The $11 x 11$ TWT has a square test section with slotted walls and runs from Mach 0.2 to 1.45. The test section is contained within a cylindrical plenum that is part of the pressure vessel. There is a three-long by five-high array of windows in each sidewall, where each window is 41 in long and 8 in high. This results in an overall viewable area of about ten by five feet. There are also three windows in both the ceiling and floor. Cameras and lamps are typically installed inside the plenum, and cooling air is often required when operating at low pressures. The 9x7 SWT has a rectangular test section with solid walls and operates a between Mach 1.55 and 2.55 . 


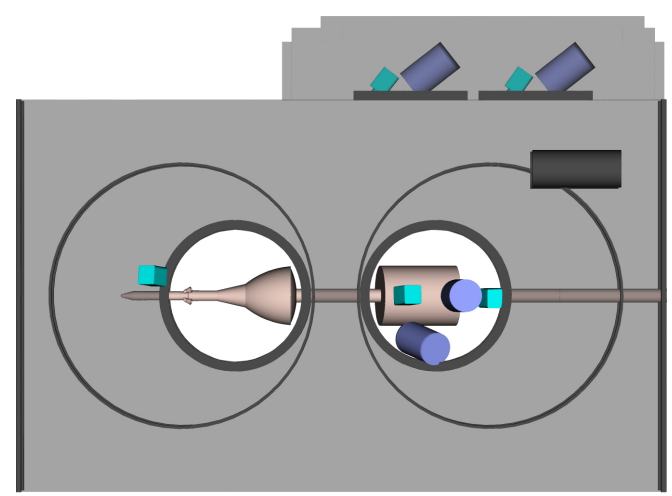

(a) $9 \times 7$ SWT, showing the lack of optical access

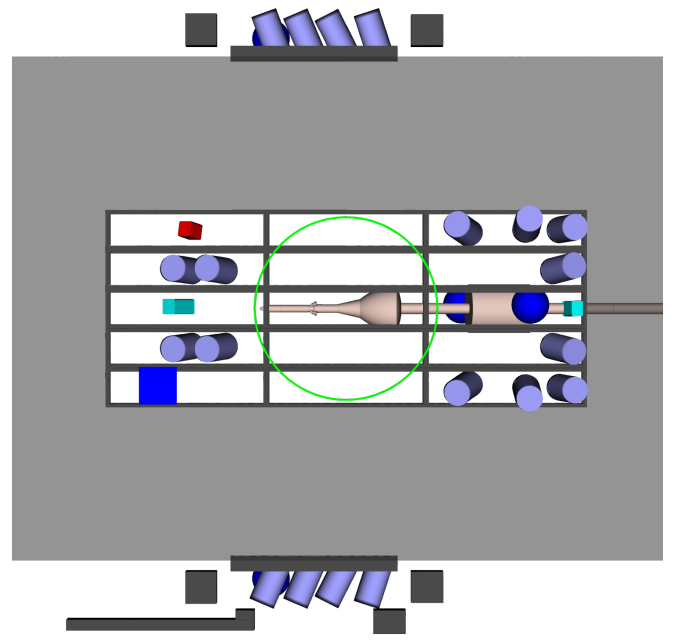

(b) $11 \times 11$ TWT

Figure 6. Virtual Images of 6\% CEV model in Unitary Plan Wind Tunnels at NASA Ames. The PSP cameras are shown in cyan, the PSP lamps in purple and the schlieren field of view is outlined in green. An IR camera is shown in red and facility cameras are shown in blue. The model is shown in PSP pink. Flow is from left to right.

Optical access for PSP is poor. In each side wall there are two 28 in diameter circular windows, each of which is eccentrically mounted in a five foot diameter turntable. These windows were designed for use with a schlieren system. Because the windows are eccentric, rotating the turntable displaces the centers of the windows making different parts of the flow available for schlieren imaging. The 9x7 SWT also has two 22 in $\mathrm{x} 11$ in windows in the ceiling, located 7 in from the tunnel centerline. There are no windows in the floor because of the sliding nozzle block which changes the Mach number of the supersonic flow. The walls and windows are part of the pressure vessel; thus, unlike in the 11x11 TWT, cameras and lamps can be mounted in an easily accessible, ambient environment.

In the 11x11 TWT, the PSP system consisted of 40 high-intensity UV LED lamps from Innovative Scientific Solutions Inc (ISSI) and eight cameras, each with 4 megapixel sensors and $12 \mathrm{~mm}$ lenses. Eight cameras were also used in the 9x7 SWT; however, because of limited window space, there was room for only $14 \mathrm{UV}$ lamps.

\section{IV.C. Virtual Image Use for CEV 26-AA PSP}

Virtual imaging was used to plan the layout of cameras and lamps and select lens focal lengths for both the 11x11 TWT and SST portions of the test. Full coverage of the model and even illumination over the full range of model angles $\left( \pm 15^{\circ}\right.$ of pitch and $\pm 10^{\circ}$ of yaw) and service module positions and angles (1.2 model diameters downstream, $\pm 5^{\circ}$ of pitch) were critical. In addition, the placement of the lamps and cameras could not interfere with the schlieren system, which was operated simultaneously, in either tunnel nor could the cameras and lamps lie in the same window pane, which could increase noise in the PSP images. Figure 6 shows the proposed initial set up of the optical systems in both tunnels. 


\section{IV.C.1. Model Illumination}

In order to accurately model the illumination of the PSP lamps, the illumination intensity from one lamp was measured as a function of distance from the lamp and angle from the lamp centerline (Figure 7 on the previous page). The normalized data show a Gaussian fall-off in intensity as the beam spreads laterally and an inverse-square fall-off in intensity in the direction of propagation. The Gaussian curve-fit shown in the figure was used to model the illumination intensity from all lamps. This allowed calculation the intensity of light that would fall on each part of the model, which would help determine the best location of the lamps to obtain the most even illumination of the model.

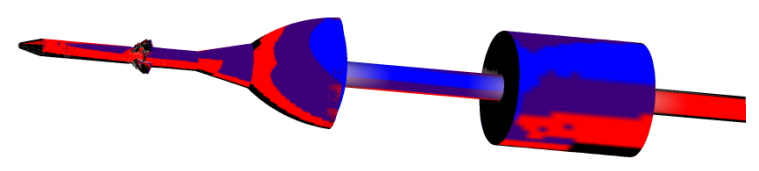

(a) Port

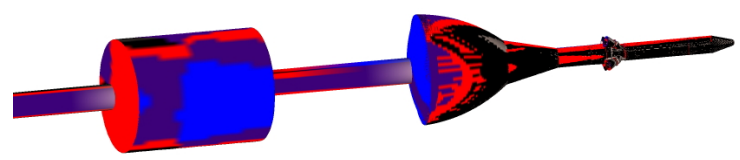

(c) Starboard

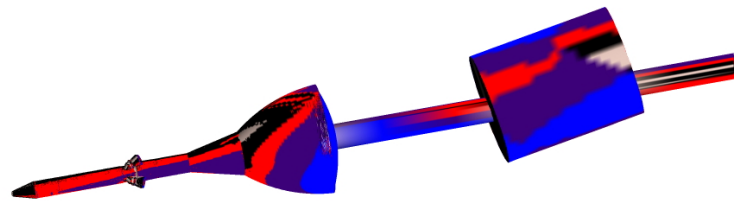

(b) Top

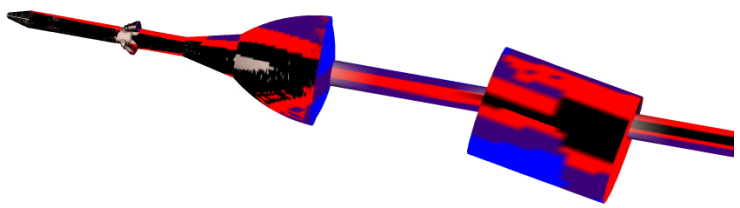

(d) Bottom

Figure 8. Model illumination in the 9x7 SWT, with the old tunnel windows. Model at $\alpha=-10^{\circ}$ and $\beta=5^{\circ}$, shaded with colors corresponding to how bright the PSP lights will be on the model. Blue is maximum light, purple is maximum light minus 1 stop, red is minus 2 stops, black is minus 3 stops and PSP pink means no light hits the model.

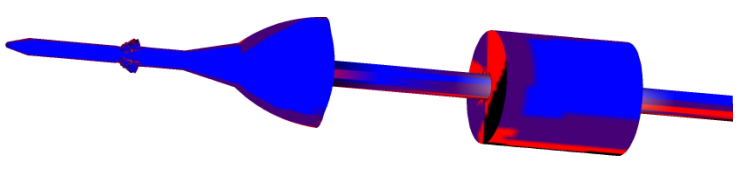

(a) Port

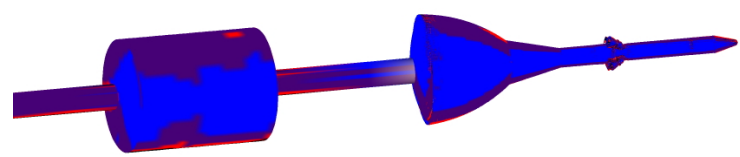

(c) Starboard

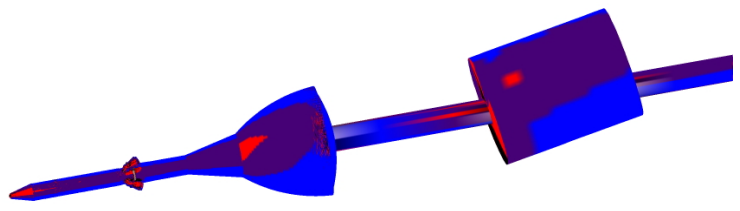

(b) Top

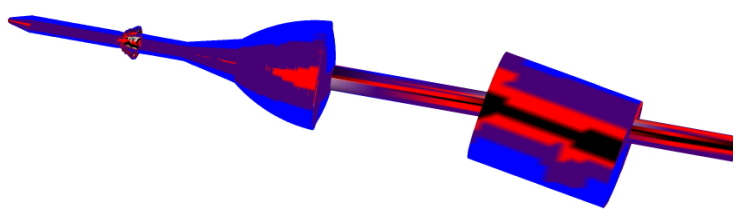

(d) Bottom

Figure 9. Model Illumination in the 9x7 SWT with the new tunnel windows. Model at $\alpha=-10^{\circ}$ and $\beta=5^{\circ}$, shaded with colors corresponding to how bright the PSP lights will be on the model. Color scheme is the same as Figure 8 .

The illumination intensities for the 9x7 SWT are shown in Figure 8 for a single model attitude. Model shading shows the intensity of the incident light in $f$-stops. Figure 8 shows that the bottom and top of the model are not well lit and the illumination intensity spans four stops. This was not an ideal setup for PSP, but was the best design that would accommodate both PSP and schlieren without modifying the tunnel. The lack of windows also made it impossible to keep the lamps and cameras in separate windows. Similar virtual images were generated for the extreme model attitudes.

Virtual imaging showed that the illumination of the model in the 9x7 SWT could be significantly improved by adding small circular windows between web structures in the turntables that supported the large schlieren windows. Figure 9 shows the intensity of the incident light using the new windows, which is more uniform than without the additional windows. On the basis of this analysis, these new windows were added as shown in Figure 10 on the next page. 


\section{IV.C.2. Camera Coverage}

Vinci was used to view the model from the point of view of all the cameras over the full range of model attitudes to make sure that all surfaces of the model were always visible in at least one camera. This was done in two steps. First, the cameras were set so that the model could be seen in all cameras for one angle. Secondly, images were created spanning the range of model attitudes that would be seen in testing to ensure the surface of the model never went outside of the FOV of any camera and that each point on the model was visible to at least one camera at an angle no greater than $70^{\circ}$. Figure 11 shows virtual images in the ARC TWT. Figure 11(a) shows the model at extreme angles as seen by one camera and Figure 11(c) shows the model at one angle as seen by four of the eight cameras. Images from the test are shown in Figures 11(b) and $11(\mathrm{~d})$ for comparison. The virtual images correctly

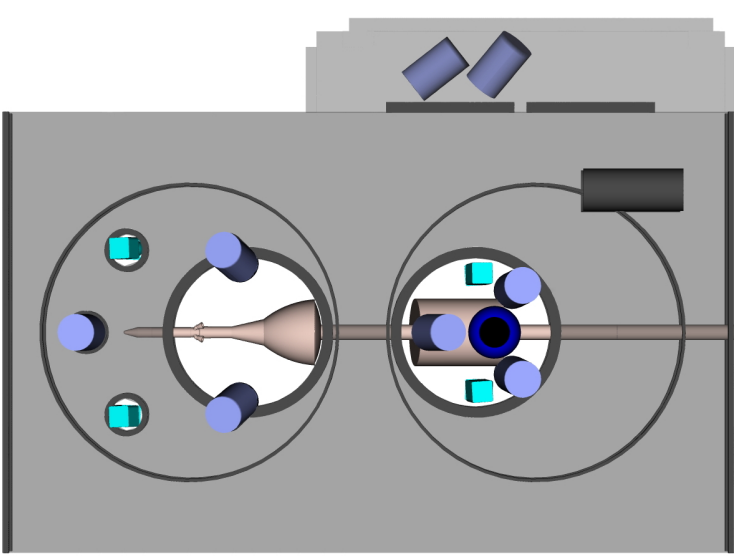

Figure 10. 9x7 SWT with new windows predicted the view from the installed cameras. An undesirable characteristic of this layout is that lamps on the opposite side of the test section appear in the images of all cameras. This problem was mitigated by adding filters to the cameras that blocked light from the lamps.

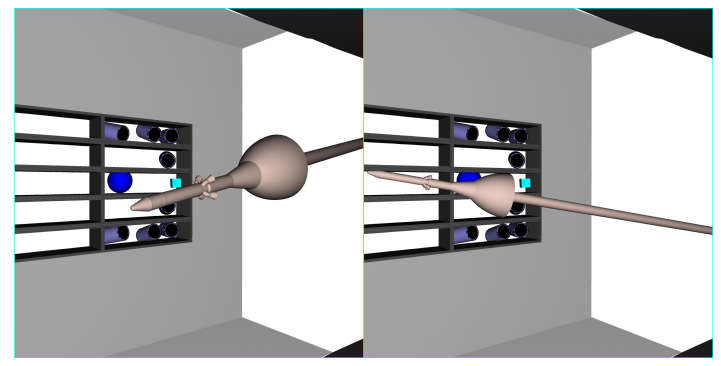

(a) Camera A at $\alpha=-10^{\circ}, \beta=10^{\circ}(\mathrm{L})$, and $\alpha=10^{\circ}$, $\beta=-10^{\circ}(\mathrm{R})$, virtual images

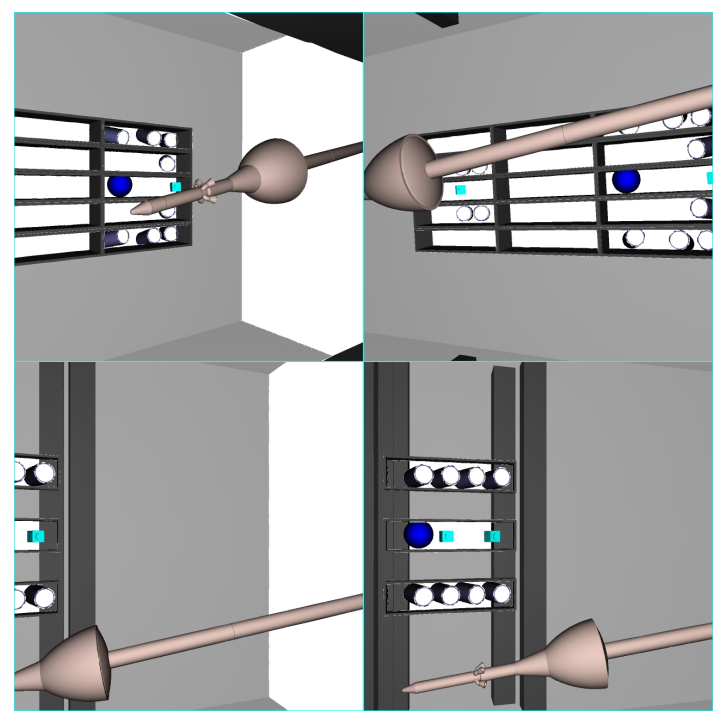

(c) Camera A, B, C, D at $\alpha=-10^{\circ}$ and $\beta=10^{\circ}$, virtual images

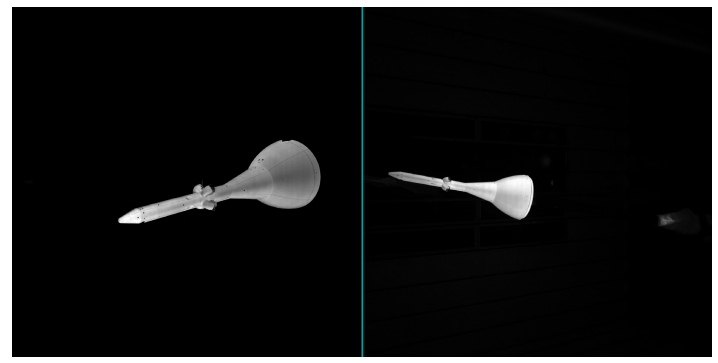

(b) Camera A at $\alpha=-10^{\circ}, \beta=10^{\circ}(\mathrm{L})$, and $\alpha=10^{\circ}$, $\beta=-10^{\circ}(\mathrm{R})$, test images

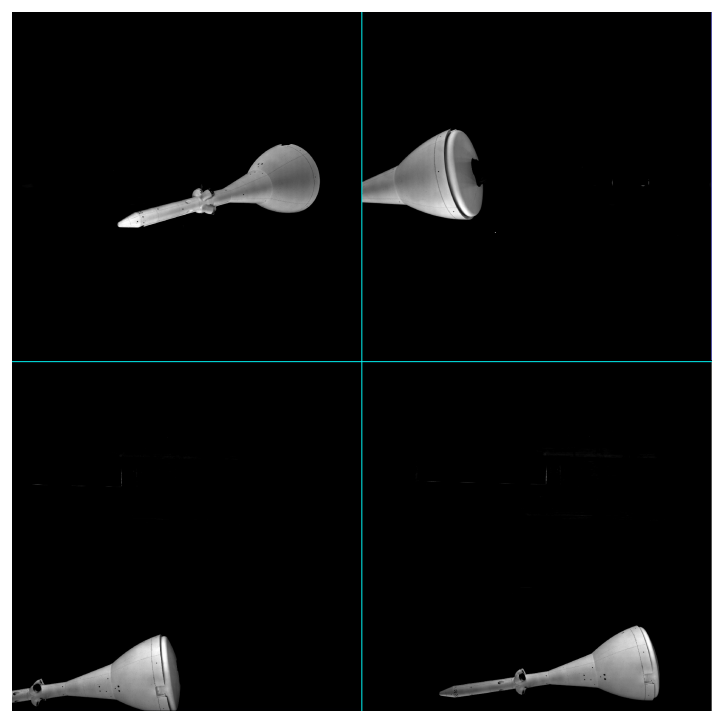

(d) Camera A, B, C, D at $\alpha=-10^{\circ}$ and $\beta=10^{\circ}$, test images

Figure 11. Virtual and real images from 26-AA in the 11x11 TWT showing that Vinci accurately predicted camera locations that fit test requirements 


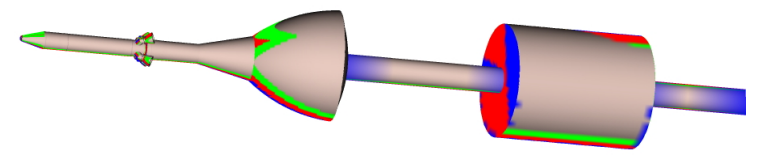

(a) Port

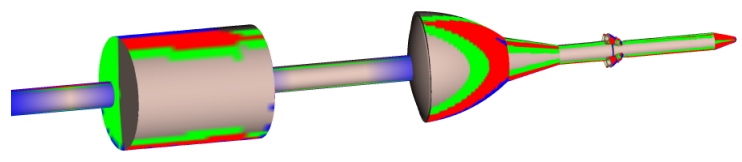

(c) Starboard

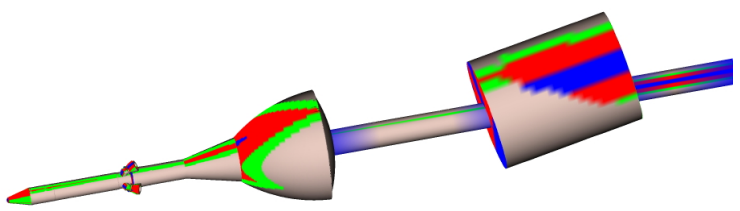

(b) Top

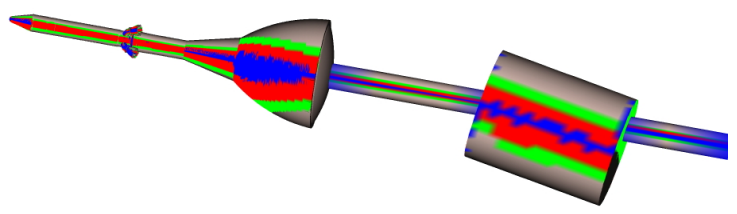

(d) Bottom

Figure 12. Model visibility in $9 \times 7 \mathrm{SWT}$ with the old tunnel windows. Model at $\alpha=-10^{\circ}$ and $\beta=5^{\circ}$, shaded with colors corresponding to best the angle of incidence from the cameras with that sport on the model in view. PSP pink corresponds to an angle of incidence of less than $60^{\circ}$, green between $60^{\circ}$ and $70^{\circ}$, red greater than $70^{\circ}$, and blue not visible.

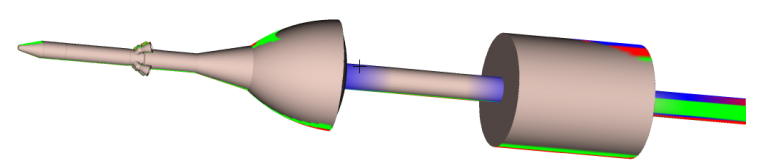

(a) Port

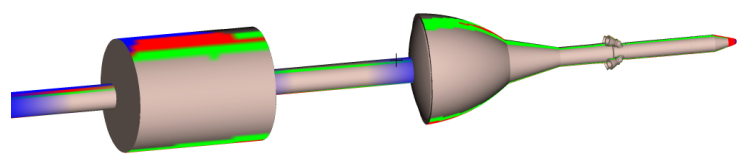

(c) Starboard

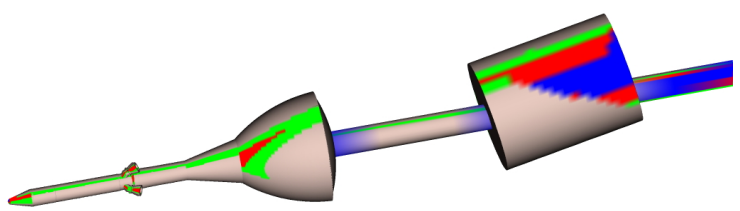

(b) Top

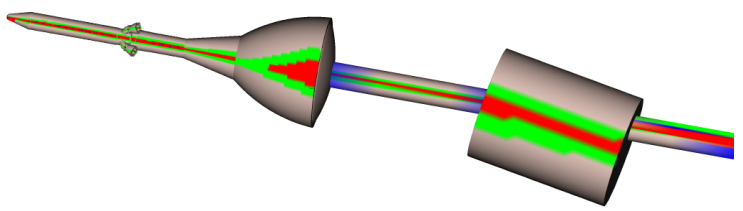

(d) Bottom

Figure 13. Model visibility in 9x7 SWT with the new tunnel windows. Model at $\alpha=-10^{\circ}$ and $\beta=5^{\circ}$, shaded with colors corresponding to best the angle of incidence from the cameras with that spot on the model in view. Color scheme is the same as in Figure 12.

In the 9x7 SWT, the visibility of the lower surface of the model was quite poor without additional windows (Figure 12). Adding windows allowed repositioning the cameras to match previous test setups and improved visibility of the lower surface, as shown in Figure 13.

\section{CRM}

The Common Research Model (CRM) was a generic, commercial low-wing transport that was designed to be tested in several wind tunnels, including the $11 \times 11$ TWT, where measurements would be made that could be used to validate CFD codes. In the test at Ames, PSP was used to measure the surface pressures on one half of the model and PIV was used to measure the flow in three different cross-stream planes on the same side as the PSP. Virtual imaging was used to plan both the PSP and PIV systems. The PSP system was designed first, and, compared to 26-AA, was an easy setup because measurements were required only on half (port side) of the model and the main constraint was to not interfere with the PIV system. Therefore, in this section only planning the PIV system is described.

\section{V.A. Particle Image Velocimetry}

Particle Images Velocimetry is an optical technique that measures the displacements of seed particles in a flow as they pass through a sheet of laser light. For each measurement, the laser is pulsed twice in rapid succession, where the time between pulses is less than the time required for most particles to traverse the 
thickness of the sheet (usually $2-3 \mathrm{~mm}$ ), and images of light scattered by the particles are acquired by cameras that are synchronized to the laser pulses. The image-plane displacements of particles between the first and second pulses are measured by cross correlating image data from the first and second pulses in small interrogation windows distributed across the image. The relationship between image and object space is established by calibration. For three-dimensional measurements, simultaneous data from a second camera that images the same particles from a different direction are also required. Stereo geometry is used to compute spatial displacements from the image-plane displacements measured in images from both cameras. The measurement uncertainty in the direction toward the baseline between the cameras is smallest when the angle between the optical axes of the cameras is $90^{\circ}$.

For transonic and supersonic flows, where there are abrupt changes in flow velocity at shock waves, PIV particles are typically $0.3-1 \mu \mathrm{m}$ in diameter, a range in which the scattered light intensity can be described by Mie scattering. In the Mie-scattering regime, the scattered light intensity depends on the particle sizes and on the direction in which the light is scattered: light scattered in the same direction as the incident laser beam (forward scatter) is much brighter than light that is scattered back toward the laser (back scatter) or light that is scattered laterally to either side. Therefore, for PIV measurements in transonic and supersonic flows where particles must be small, scattered light must be collected by the cameras in forward scatter: backscattered light is usually far too dim.

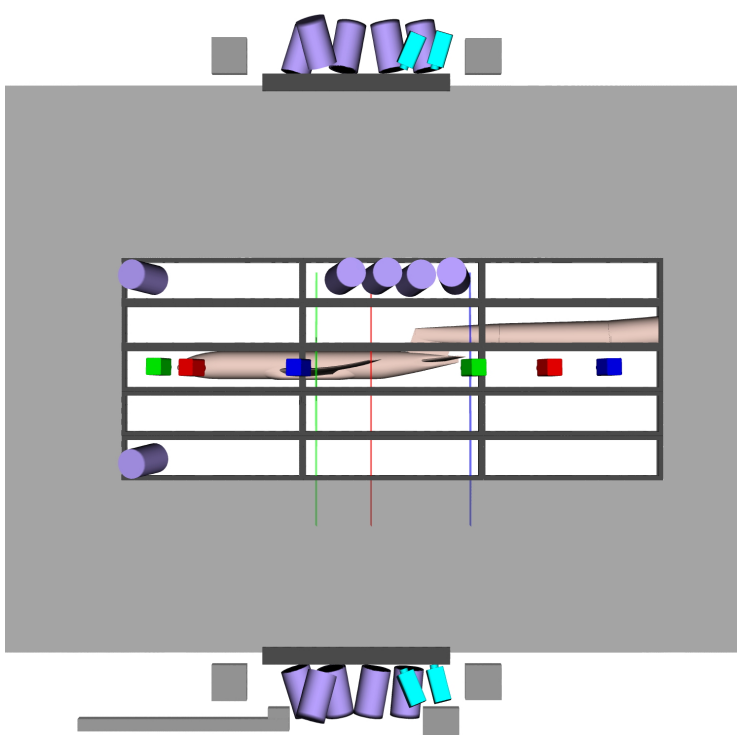

(a) Side view

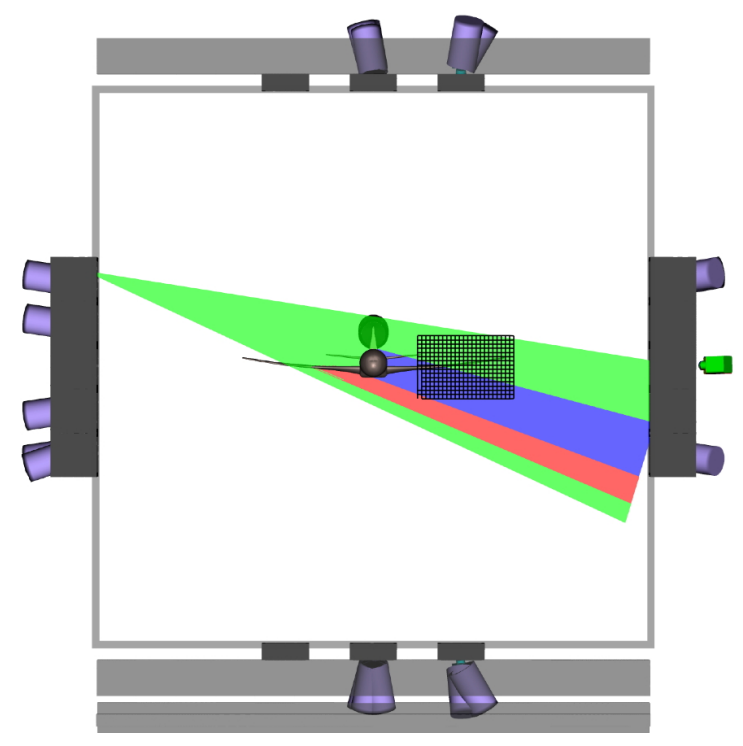

(b) Downstream

Figure 14. Layout of the PSP and PIV systems in the 11x11 TWT for CRM. The three laser locations are shown in green, red and blue with their cameras shaded accordingly. The PSP lamps are in purple and the cameras in cyan. The model is shown in PSP-pink.

\section{V.B. Wind Tunnel and Imaging System}

The regions of interest for PIV measurements were three cross-stream planes: one just upstream of the wing (to characterize the inflow); the second just downstream of the wing (to measure the wing wake, especially inboard to capture possible flow separation); and the third just downstream of the tail (to measure the wake of the horizontal stabilizer). Just as for PSP, all PIV measurements were made on the port side of the model. The ROIs were separately illuminated by a light sheet from a laser pulsing at high frequency $(1-2 \mathrm{kHz})$. A collimated laser beam ran parallel to the test section, then was directed through sheet-forming optics and across the test section by a movable mirror that controlled which ROI was illuminated. This design made it easy to switch the laser to the different ROIs with little downtime. Because the fuselage of the model blocked portions of the laser sheet and thus prevented parts of the ROIs from being illuminated, the laser sheet was projected slightly downward through the uppermost sidewall window pane, thereby moving the shadowed region downward below the wing. The ROI at each tunnel station was imaged symmetrically in forward scatter by two high-speed cameras, one upstream and one downstream of each of the vertical laser planes. 


\section{V.C. Virtual Image Use for CRM}

Vinci was used to lay out the PIV system and verify that the ROIs would be visible to the cameras. Figure 14 on the previous page shows the proposed setup. The large streamwise extent of the sidewall windows in the ARC TWT allowed placing each pair of cameras symetrically at angles relative to the laser sheet of $30-40^{\circ}$, which is a good compromise between maximum sensitivity to the spanwise velocity $\left( \pm 45^{\circ}\right)$ and signal brightness (maximum at $0^{\circ}$ ). Figure 15 shows virtual images from the most downstream camera. A grid is included that shows where measurements were desired in the most downstream measurement plane. Portions of ROIs in all three measurement planes were either not illuminated because they lay in the shadow of the fuselage (or sting at the most downstream station) or could not be seen by one of the cameras because the view was blocked by the port wing. In particular, with this setup, measurements were not possible in the inboard region downstream of the wing. It was important that this information was available in advance of the test so that the customer would have realistic expectations of what could be accomplished.

\section{Conclusion}

Virtual imaging has become an indispensable tool for planning image-based measurements in production wind tunnels at NASA-ARC. It has facilitated the planning of many complex measurement systems long before testing begins, thereby reducing occupancy time and cost and resulting in more nearly optimum systems than would otherwise have been possible. Virtual imaging is particularly valuable in planning tests where the placement of cameras and light sources is constrained by optical access through test-section windows. The periscope for the LRTA UH-60 Airloads test in the NFAC was designed using only information provided by virtual imaging and would not have been attempted without that capability. Virtual imaging was used to optimize the layout of many lamps and cameras for PSP measurements in 26-AA in both the 9x7 SWT and 11x11 TWT and to successfully advocate adding ports in the turntables of the 9x7 SWT that improved both uniformity of illumination and camera coverage of the model. Virtual imaging also provided advance knowledge of where PIV measurements were possible for the CRM test in the $11 \times 11$ TWT.

\section{Acknowledgments}

Special thanks to JT Heineck, whose idea it was to use RBOS to locate vortices in the UH-60 Airloads test, for guidance on the periscope design and PIV system layout. Thanks also to Louise Walker who spent countless hours creating the backgrounds and installing equipment based on these visualizations.

\section{References}

${ }^{1}$ Schairer, E. T.; Heineck, J. T.; Walker, S. M.; Yaste, D. M.; "Predicting Camera Views for Image-Based Measurements in Wind Tunnels," AIAA 2005-1349, 43rd AIAA Aerospace Sciences Meeting and Exhibit, 2005.

${ }^{2}$ Heineck, J.T.; Schairer, E.T.; and Walker, S.M.; "PIV Measurements of Flow Past the Space Shuttle Ascent Configuration in the NASA Ames 9- by 7-Foot Supersonic Wind Tunnel," 6th International Symposium on Particle Image Velocimetry, Pasadena, CA, Sept 21-23, 2005.

${ }^{3}$ Jordan, J.D.; Watkins, N., Fleming, G.A.; Leighty, B.D.; "Rapid Technology Assessment via Unified Deployment of Global Optical and Virtual Diagnostics," International Congress on Instrumentation in Aerospace Simulation Facilities, 2003.

${ }^{4}$ Walatka, P. P.; Clucas, J.; McCabe, K.; and Plessel, T.; "FAST User Guide," RND-92-013, June, 1992.

${ }^{5}$ Schairer, E.T.; and Heineck, J.T.; "Photogrammetric Recession Measurements of Ablative Materials in Arcjets", Measurement Science and Technology, 21, 025304, 2010.

${ }^{6}$ Abdel-Aziz, Y.I.; and Karara, H.M; "Direct Linear Transformation from Comparator Coordinates into Object-Space 
Coordinates," Proceedings of the Symposium on Close-Range Photogrammetry, Urbana, Illinois, Jan. 1971.

${ }^{7}$ Norman, T. R.; Shinoda, P. M.; Peterson, R. L. and Datta, A.; "Full-Scale Wind Tunnel Test of the UH-60A Airloads Rotor, American Helicopter Society 67th Annual Forum, Virginia Beach, VA, May 2011, in preparation.

${ }^{8}$ http://www.arnold.af.mil/library/factsheets/factsheet.asp?id=13107

${ }^{9}$ Meier, G.E.A., "Hintergrund Schlierenmeßverfahren," Deutsche Patentanmeldung DE 19942856 A1, 1999.

${ }^{10}$ Heineck, J. T.; Schairer, E. T.; Kushner, L. K; Walker,L. A.; "Retroreflective Background Oriented Schlieren (RBOS)

As Applied to Full-Scale UH-60 Blade Tip Vortices", American Helicopter Society Aeromechanics Specialists' Conference, San Francisco, CA Jan. 20-22, 2010.

${ }^{11}$ Mikhail, E. M., Introduction to Modern Photogrammetry, John Wiley \& Sons, Inc, New York, 2001.

${ }^{12}$ Meyn, L.A.; and Bennett, M.S.; "Application of a Two Camera Video Imaging System to Three-Dimension Vortex Tracking in the 80- by 120-Foot Wind Tunnel," AIAA 93-3439, AIAA Applied Aerodyanmics Conference, Monterey, CA, Aug. 9-11, 1993.

${ }^{13}$ Ross, James C.; "Aerodynamic Testing in Support of Orion Spacecraft Development", AIAA 2007-1004, 45th AIAA Aerospace Sciences Meeting and Exhibit, Reno, NV, January 2007.

${ }^{14}$ Bell, J. H.; Schairer, E. T.; Hand, L. A.; Mehta, R. D.; "Surface Pressure Measurements Using Luminescent Coatings," Annual Review of Fluid Mechanics, Vol. 33, 2001, pp. 155-206.

${ }^{15}$ http://windtunnels.arc.nasa.gov/ 